\title{
Protein Localization in Electron Micrographs
}

Shuo $\mathrm{Li}^{1}{ }^{1,2}$, Sumana Raychaudhuri² ${ }^{2}$, Shigeki Watanabe ${ }^{2,3}$

1. Department of Biochemistry and Molecular Biology, Johns Hopkins Bloomberg School of Public Health, Baltimore, MD, USA

2. Department of Cell Biology, Johns Hopkins University, School of Medicine, Baltimore, MD, USA

3. Solomon H. Snyder Department of Neuroscience, Johns Hopkins University, School of Medicine, Baltimore, MD, USA

Understanding cellular function requires precise localization of proteins in their subcellular context. Traditionally, two approaches have been used: electron microscopy and light microscopy. Electron microscopy resolves cellular structures at the nanoscale. Gold-labeled antibodies are routinely used to localize proteins in electron micrographs. However, this approach has many drawbacks. First, the protein epitopes sometimes are not preserved during the dehydration and fixation steps of sample preparation for electron microscopy. Second, epitopes in plastic embedded sample may not be recognized. Third, the morphology is compromised. Fourth, nonspecific background labeling is high. Thus, although this approach allows precise localization of proteins to subcellular structures, it is not reliable.

In contrast, light microscopy can visualize essentially any proteins if they can be genetically tagged or labeled by antibodies. However, protein localization by this approach is limited by two factors: the diffraction limit and subcellular context. Super-resolution microscopy pinpoints the locations of proteins with nanometer resolution $[1,2,3]$. This overcomes the former limitation, but not the latter: proteins are only seen on a dark background.

Electron microscopy delineates subcellular structures, while super-resolution microscopy allows precise localization of proteins. To unite the advantages of these two approaches, we developed a protocol that preserves fluorescence in specimens prepared for electron microscopy to localize proteins using correlative super-resolution and electron microscopy [4] (Fig. 1).

The correlative microscopy approach allows precise localization of proteins in their subcellular context. However, the alignment of super-resolution images and electron micrographs is extremely difficult due to irradiation damage in an electron microscope, imposing uncertainty in the localization. In addition, super-resolution imaging is typically time-consuming. Thus, a more robust and direct method is needed. Several approaches have been developed to mark proteins of interest with electron dense materials in recent years, notably metallothionein and diaminobenzidine. Metallothionein is a cysteine-rich small (61aa) metal binding protein that binds up to 20 gold atoms and has been used for localization of proteins in electron micrographs [5]. However, the method is limited to abundant proteins, and incubation with a toxic heavy metal compromises cell health. For diaminobenzidine, modified ascorbate peroxidase and singlet oxygen generator, miniSOG, are used to oxidize diaminobenzidine to form precipitates. This diaminobenzidine reaction product in turn reacts with osmium tetroxide to enhance the electron density around the protein of the interest [6,7]. However, these techniques are limited by diffusion of diaminobenzidine and abundance of proteins. We are currently developing a novel approach to overcome these issues and pinpoint the location of proteins in electron micrographs (Fig. 2) [8]. 


\section{References:}

[1] SW Hell, Science 316 (2007), p. 1153.

[2] E Betzig el al, Science 313 (2006), p. 1642

[3] ST Hess et al,, Biophys. J. 91 (2006), p. 4258.

[4] S Watanabe et al, Nat. Methods 8 (2011), p. 80.

[5] C Risco et al, Struct. Lond. Engl. 199320 (2012), p. 759.

[6] J Zhang et al, Nat. Rev. Mol. Cell Biol. 3 (2002), p. 906.

[7] JD Martell, et al, Nat. Biotechnol. 30 (2012), p. 1143.

[8] The authors acknowledge the funding from Johns Hopkins Start-up Funds, Johns Hopkins Discovery award, NSF (1727271)

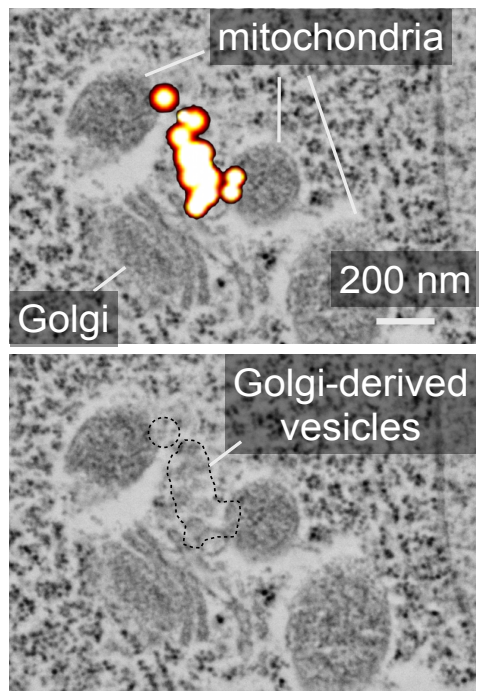

Figure 1. Example micrographs showing clathrin-tdEos localization on Golgi-derived vesicles by correlative microscopy
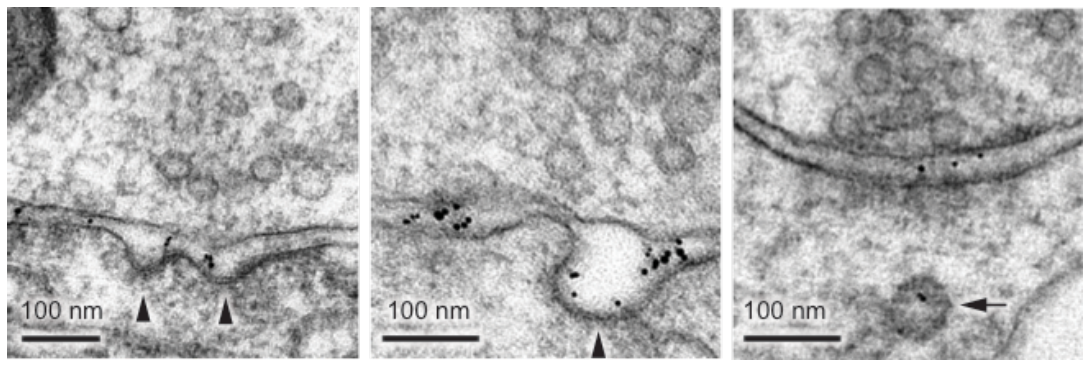

Figure 2. Representative micrographs showing tagged AMPA receptors at post-synaptic terminals. Magnification, 93000X 